\title{
GA-Optimized Multivariate CNN-LSTM Model for Predicting Multi-channel Mobility in the COVID-19 Pandemic
}

\author{
Harya Widiputra ${ }^{1 *}$ \\ ${ }^{1}$ Faculty of Information Technology, Perbanas Institute, Jakarta, Indonesia
}

\begin{abstract}
The primary factor that contributes to the transmission of COVID-19 infection is human mobility Positive instances added on a daily basis have a substantial positive association with the pace of human mobility, and the reverse is true. Thus, having the ability to predict human mobility trend during a pandemic is critical for policymakers to help in decreasing the rate of transmission in the future. In this regard, one approach that is commonly used for time-series data prediction is to build an ensemble with the aim of getting the best performance. However, building an ensemble often causes the performance of the model to decrease, due to the increasing number of parameters that are not being optimized properly. Consequently, the purpose of this study is to develop and evaluate a deep learning ensemble model, which is optimized using a genetic algorithm (GA) that incorporates a convolutional neural network (CNN) and a long short-term memory (LSTM). A $\mathrm{CNN}$ is used to conduct feature extraction from mobility time-series data, while an LSTM is used to do mobility prediction. The parameters of both layers are adjusted using GA. As a result of the experiments conducted with data from the Google Community Mobility Reports in Indonesia that ranges from the beginning of February 2020 to the end of December 2020, the GA-Optimized Multivariate CNN-LSTM ensemble outperforms stand-alone CNN and LSTM models, as well as the non-optimized CNN-LSTM model, in terms of predicting human movement in the future. This may be useful in assisting policymakers in anticipating future human mobility trends.
\end{abstract}

\section{Keywords:}

Deep Learning Ensemble; Convolutional Neural Network; Long Short-term Memory; Mobility Prediction;

Genetic Algorithm;

COVID-19.

\section{Article History:}

$\begin{array}{llll}\text { Received: } & 12 & \text { June } & 2021 \\ \text { Revised: } & 08 & \text { September } & 2021 \\ \text { Accepted: } & 19 & \text { September } & 2021 \\ \text { Published: } & 01 & \text { October } & 2021\end{array}$

\section{1- Introduction}

People are traveling more than ever before because to advancements in transportation technologies. Although the increased connectivity between distant nations has numerous advantages, it also presents a significant danger to disease management and prevention [1]. When sick people go to areas where their contagions aren't present, they may unintentionally spread their illnesses to locals, resulting in disease outbreaks [2]. This has happened many times in history; recent instances include the SARS epidemic in 2003, the H1N1 influenza pandemic in 2009, and, most significantly, the continuing Coronavirus disease (COVID-19) pandemic [3, 4]. COVID-19 is a recently identified coronavirus that causes an infectious illness. When an infected individual coughs or sneezes, the COVID-19 virus spreads mainly via saliva droplets or nasal discharge $[4,5]$. As a result, being well-informed on the COVID-19 virus, the illness it causes, and how it transmits is the greatest approach to avoid and limit transmission [6, 7].

Respectively, over the last several decades, scientists have spent a lot of time looking at how human movement impacts disease transmission [8-10]. Previous research has shown that a disease has a propensity to travel to densely populated metropolitan regions before spreading to more rural places, and then to all nearby sites. The timing of the infection's initial entrance, which is related to the landscape of human movement defined by relative attraction, may

* CONTACT: harya@perbanas.id

DOI: http://dx.doi.org/10.28991/esj-2021-01300

(C) 2021 by the authors. Licensee ESJ, Italy. This is an open access article under the terms and conditions of the Creative Commons Attribution (CC-BY) license (https://creativecommons.org/licenses/by/4.0/). 
capture the infection's diverse spread and therefore it is learnt that in the time and geographical transmission of infectious illnesses, human movement is critical [7, 9, 10]. Consequently, countries all across the globe reacted to the COVID-19 epidemic by imposing what may possibly be the most comprehensive set of state-led movement and activity restrictions in human history. In this regard, Lai et al. and Chang et al. conducted research to see how successful these strategies were in different nations. According to their findings, restricting societal movement in a seven-to-fourteen-day pattern was most successful in controlling the COVID-19 pandemic and should be regarded as important countermeasures to fight the pandemic effectively $[11,12]$.

Referring to the various facts that have been conveyed regarding the relationship between the movement of people in an area to the spread of COVID-19 infection which is very significant [8-12], it can be concluded that if the Government has the ability to predict trends in community movements, then this can be used to formulate policies for handling spread of COVID-19 infection. However, the challenge that arises is to be able to define a time-series data prediction model that has good performance and is reliable. As is well known, a stand-alone time-series data prediction model often has inconsistent performance, so building an ensemble which is a combination or integration of several models is often the main solution. Nevertheless, building an ensemble also often results in a decrease in overall model performance, due to the increased complexity of the model and the increasing number of parameters that are often not optimized. In addition, the time-series data prediction models that are commonly used often suitable only of the process of predicting univariate time-series. Therefore, these models are not suitable when the problem is to predict movement of multivariate time-series data such as data on community movements that occur not only in one regional group but are spread over several groups or locations.

The outlined problems above, namely the decrease in the performance of the ensemble model caused by un-optimized parameters and the need for a time-series data prediction model that is able to handle multivariate data, are the two main challenges solved in this study. Accordingly, this research presents a deep learning ensemble model architecture, whose parameters are fine-tuned, with prediction on multivariate time-series data as its major contribution. Furthermore, to confirm the performance of the proposed model, a performance test is carried out by utilizing real data on the movement of people in an area during the period of spreading COVID-19 infection in Asia by utilizing data published in Google Community Mobility Reports.

The first section of this article discusses the backdrop for conducting this study as well as the objectives that must be accomplished. Section 2 describes several outcomes from previous research that are connected to state-of-the-art timeseries data prediction approaches, as well as their relevance to the proposed model. The idea and operation of the proposed multi-channel data mobility prediction model are explained in Section 3 of this work. In addition, Section 4 discusses data, parameter settings, and assessment techniques, followed by a discussion of the outcomes from the experiments in Section 5. Finally, Section 6 presents some findings as well as a plan for future study.

\section{2- Related Works}

This section reviews some of the past studies that have utilized deep learning models which have shown to be effective and are extensively used in time-series data prediction procedures. Convolutional Neural Networks and Long Short-Term Memory are two deep learning models that are frequently employed for time-series data prediction. Additionally, a description of the ensemble structure of the two models that are frequently utilized to take use of each model's benefits is also included in this section. Eventually, discussion regarding the application of optimization techniques for performance enhancement, particularly in the ensemble model is also given in the final part of the section.

\section{2-1-Convolutional Neural Network}

The convolutional neural network $(\mathrm{CNN})$ is a deep learning model that is widely used in computer vision work and has also been used to acoustic modeling for automatic voice recognition (ASR) [13]. Because the patterns of connections between neurons mirror the structure of an animal's visual cortex, CNN are motivated by biological processes [14, 15]. Image and video recognition, recommendation systems, image classification, medical image analysis, and natural language processing are all areas where $\mathrm{CNN}$ is utilized [13-15].

However, prior research has shown that $\mathrm{CNN}$ is also excellent in processing time-based flowing data, such as timeseries prediction. This is due to CNN's primary characteristic of processing multichannel input data, which makes it suitable for handling various time-series data with numerous inputs and outputs in this research [15-17]. Figure 1 depicts the basic structure of $\mathrm{CNN}$ when applied to the process of time-series prediction.

One of CNN's major benefits is its local perception and weight sharing characteristics, which may drastically decrease the number of parameters and therefore improve the learning process' efficiency. The convolutional layer and the pooling layer are the two major components of CNN in terms of structure. Each convolution layer in this instance includes multiple convolutional kernels. The essential characteristics of the data are retrieved after the convolution process at the convolution layer, which is followed by an increase in the feature dimensions. A layer of integration is introduced to 
address this issue and decrease the load on the training process, with the primary goal of decreasing the number of features extracted before ultimately delivering the final output.

As previously stated, CNN has been extensively investigated as a solution to the issue of time-series prediction, especially in financial contexts. For instance, Chong, Han and Park published a comprehensive review of the application of deep learning networks, such as CNN, for stock market forecasting and prediction in 2017 [18]. Deep neural networks will extract more information from the residuals of the autoregressive model and enhance prediction accuracy, according to the study's empirical findings. While $\mathrm{CNN}$ is most often used for image recognition and feature extraction, according to Shih, Sun and Lee experimental results from 2019, it may also be utilized for time-series prediction even though the forecasting accuracy is considerably uncompetitive [19]. Hoseinzade and Haratizadeh (2019) have suggested a CNNbased method in 2019 that can be used to extract characteristics from a range of data sources, including various marketplaces, in order to forecast their future [20].

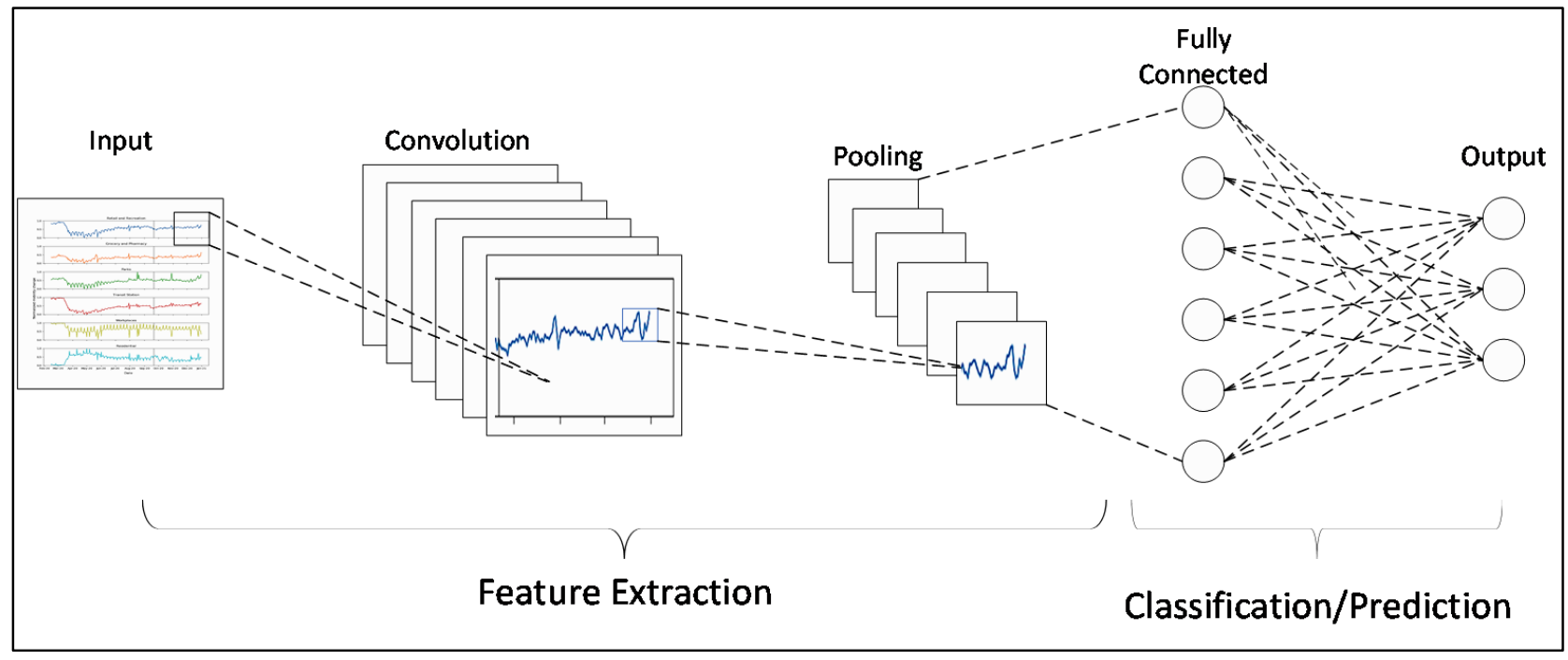

Figure 1. Illustration of feature extraction and prediction from time-series data by CNN when being applied on multivariate time-series data.

\section{2-2-Long Short-Term Memory}

The long short-term memory (LSTM) network is made up of layers of neurons, much like a multi-layer perceptron (MLP). To produce a forecast, input data is transmitted through the network. The LSTM, like recurrent neural network (RNN), contain recurrent connections, which means that the state from prior neuron activations from the preceding time step is utilized as background for forming an output. However, unlike traditional MLP or other RNN, the LSTM has a unique formulation that enables it to sidestep the issues that prohibit other RNN from being trained and scaled [21]. The technique's popularity stems from this, as well as the remarkable results that may be obtained.

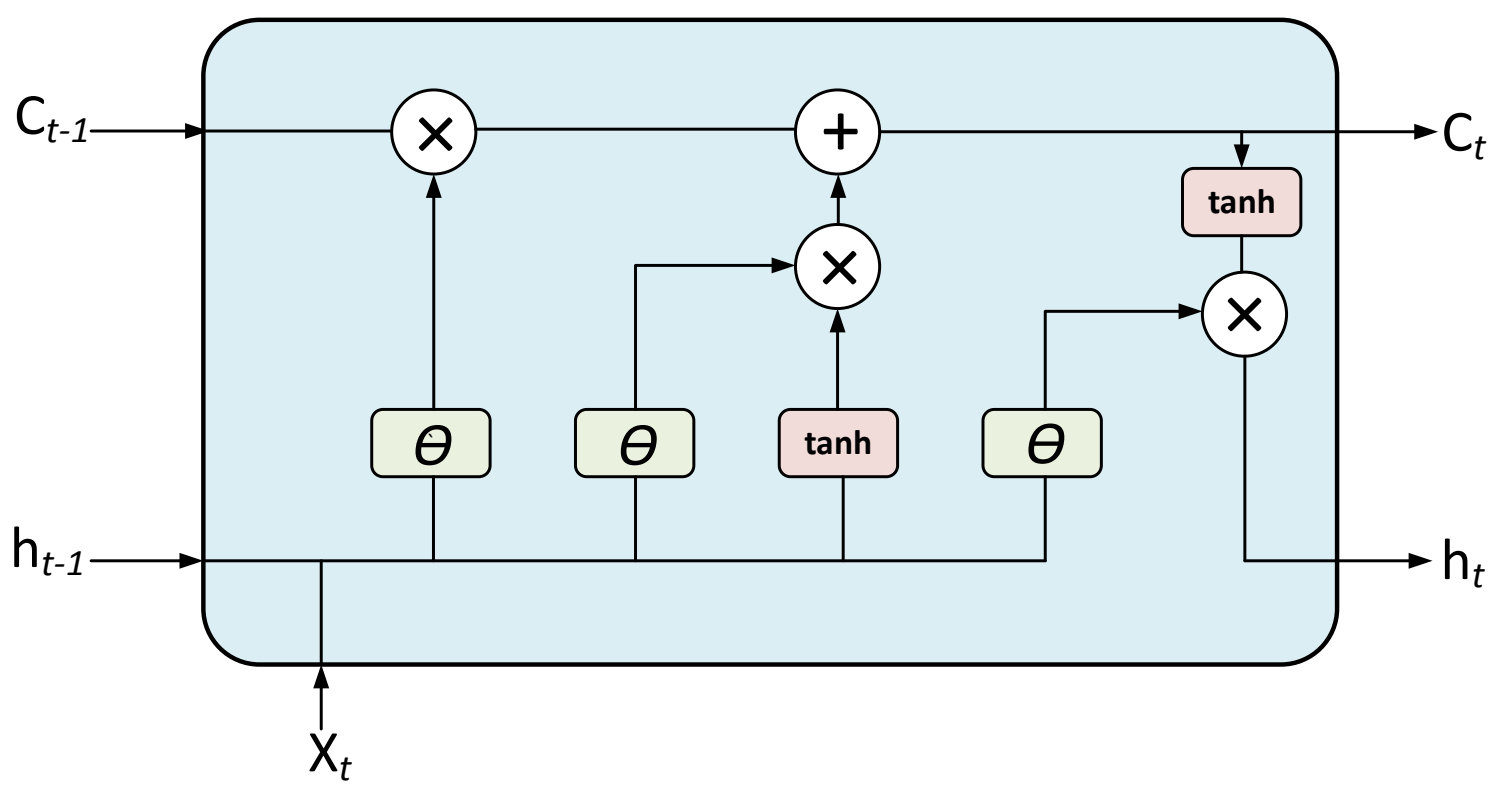

Figure 2. Basic flow of process of LSTM for time-series prediction [21]. 
The main technological issue with RNN in the past has been how to properly train them [21, 22]. Experiments have shown how difficult this was, with weight changes that soon either so tiny that they had no impact (vanishing gradients) or so big that they caused extremely significant changes or even overflow (exploding gradients). By design, LSTM circumvent this difficulty [21]. Generally, based on findings from previous works in the area of LSTM, the three main advantages of LSTM can be summarized as follows:

- Overcomes the technical issues associated with RNN training, such as disappearing and bursting gradients.

- To address the problems of long-term temporal dependence on input sequences, it has memory.

- Time step by time step, process input and output sequences, enabling variable length inputs and outputs.

The memory cell, memory block, or simply cell is the computational unit of the LSTM network. When discussing MLPs, the word neuron as a computational unit has become so entrenched that it is also often used to refer to the LSTM memory cell. Weights and gates are the components of LSTM cells and the logical structure of LSTM, is usually characterized as shown in Figure 2.

The LSTM method is excellent for classifying, sorting, and forecasting time-series dataset [14, 23, 24]. LSTM has also been proven to be capable of predicting time-series data in previous studies [23, 24]. For example, on the Tehran stock market in 2020, Nabipour et al. examined different time-series prediction methods and discovered that the LSTM delivers more accurate findings and has the greatest model fitting ability [24]. Kamalov used MLP, CNN, and LSTM to predict the stock values of four major US public companies in 2020. In terms of experimental results, these three methods outperformed comparable studies that predicted the trajectory of price change [25]. Liu and Long created a highprecision short-term forecasting model of financial market time-series in 2020, which they compared to the BP neural network, conventional RNN, and improved LSTM deep neural networks [26].

\section{2-3-Ensemble of CNN and LSTM}

Deep learning models that explore complex and unknown patterns in vast and diverse data sets may be created using both LSTM and CNN [27-29]. The aim was then to build an ensemble by combining various deep learning models, both CNN and LSTM-based in particular to overcome the problem of time-series prediction. Because prior research has shown that various models have varied capacities to detect hidden patterns in data, it is anticipated that the answers given by this ensemble approach would be stronger and more comprehensive.

A number of prior studies have been done to support the concept of creating an ensemble of CNN and LSTM to improve the performance of time-series prediction, and the results are encouraging. Lu et al., for example, presented an ensemble structure of CNN-LSTM and shown that it is successful when used to forecast the Shanghai Composite Index [29]. In addition, Mahmud and Mohammed conducted a study in 2021 on the use of deep learning algorithms for timeseries forecasting, finding that deep learning methods such as CNN and LSTM provide better prediction results with lower error levels than other artificial neural network models [30]. Furthermore, their research found that combining several deep learning models significantly increased time-series prediction accuracy.

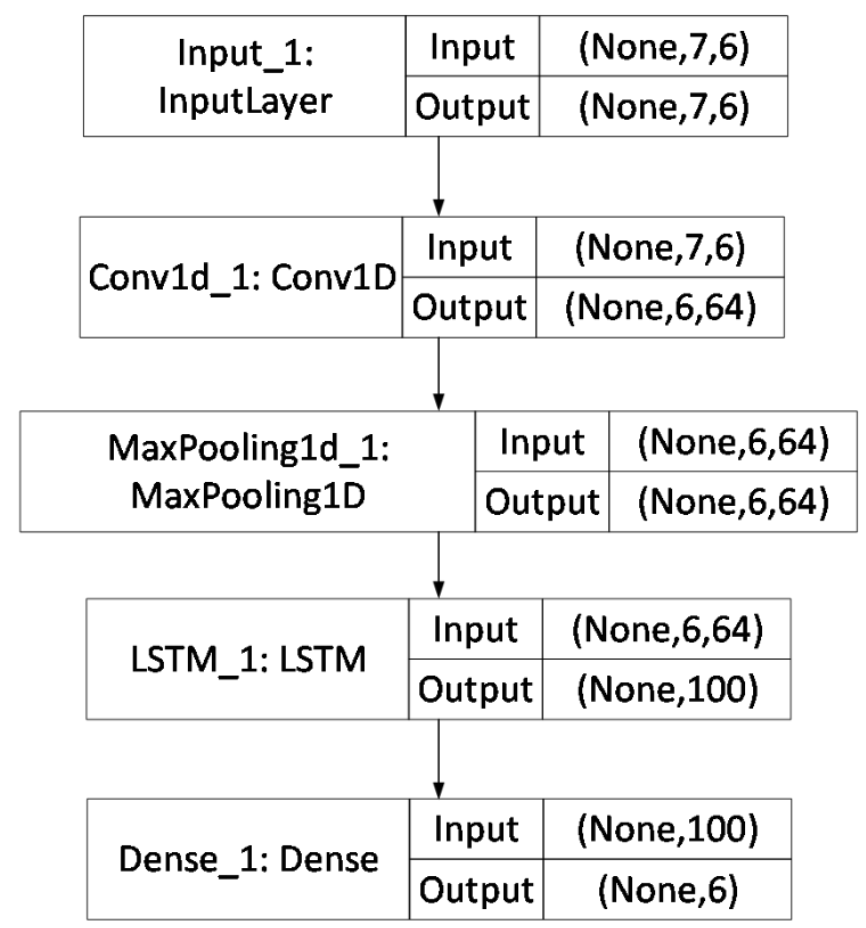

Figure 3. Basic structure of the CNN and LSTM ensemble model for time-series prediction. 
However, Mahmud and Mohammed also mentioned that CNN and LSTM's performance isn't always constant, with CNN outperforming LSTM at times and vice versa [30]. When dealing with time-series data made up of a collection of pictures, CNN seems to have a better predictive ability than LSTM, but when dealing with numerical data, LSTM looks to be superior. In conclusion, prior research shows that utilizing deep learning models like CNN and LSTM for financial time-series prediction is effective. However, when applied to numerical time-series data, CNN has worse prediction accuracy than LSTM owing to its fundamental features, which include a high point in feature extraction. When compared to CNN, LSTM has a deficit in terms of its capacity to extract the most important characteristics from a data set.

As a result, it makes sense to build a composite or ensemble model that takes use of each combination model's strengths to overcome its shortcomings and improve time-series prediction accuracy. Consequently, Figure 3 depicts the fundamental structure of the CNN and LSTM ensemble models, with the LSTM layer layered on top of the CNN layer. In this case, the input will be sent first into the CNN layer, which will extract the features, and then into the LSTM layer, which will compute the final predictions.

\section{2-4-Genetic Algorithm Optimized Ensemble}

One of the main challenges that arise when building an ensemble which is an integration of several models is the increasing number of parameters that must be determined in order to give the best results. This condition is a logical consequence, because each model certainly has several parameters and when two or more models are combined in an ensemble there is an accumulation of the total number of parameters of the entire model.

The main purpose of building an ensemble is to be able to take benefit of the various advantages possessed by each model in order to provide the best results. However, the condition of increasing the number of parameters is a challenge because if optimization is not carried out properly, the presence of the ensemble will not be able to provide better results. This is confirmed from the findings of various studies that have been done previously. As an example: Monica, Melin and Sachez who exposed that optimization is an imperative process on the ensemble of neural network architectures they developed for prediction of COVID-19 confirmed and death cases [31]; Liu, Hara and Kita who in their study have found that optimized deep learning networks is giving higher accuracy when predicting wind speed [32]; and study by Vadicharla and Sharma that explores various optimization approach which also found that ensemble of models needs to be finely tuned in order to give the best result [33].

Accordingly, an optimization procedure that runs automatically and forms part of the structure of the constructed ensemble model is considered essential in the CNN and LSTM ensemble model presented in this research. Thus, an optimization method for CNN and LSTM parameters utilizing genetic algorithm (GA) was applied in the construction of CNN and LSTM models for prediction of multi-channel community movements in this study.

GA was chosen based on the findings of past research in the area of optimization, which show that GA has excellent performance, is light on computation, and is easy to use [34,35]. Several researches in the field of optimization using GA include the following: the study by Qu et al. that combined genetic optimization with AdaBoost ensemble model for anomaly detection in buildings electricity consumption [36]; a work by Dowes, Nair and Sharma in 2021 that build an ensemble of GA and machine learning classifier for blood sample classification to diagnose COVID-19 [37]; and a study by Gao, Gao and $\mathrm{Li}$ that has found the effectiveness of GA when being applied to ensemble of CNN to recognize defects in small sample data [38].

\section{3- GA-Optimized Multivariate CNN-LSTM for Multi-channel Mobility Prediction}

\section{3-1-Research Methodology}

The research methodology used in this study is a constructive research methodology. In this methodology, the main focus of research is to develop and build an artifact, which in this case is an ensemble architecture of deep learning models that are optimized for prediction of multivariate time-series data. In addition to building an artifact, the constructive research methodology also focuses on testing and evaluating the artifact in question by conducting trials using real data and comparing the results obtained from other techniques. In general, the research methodology used in this study is depicted in the flowchart in Figure 4.

As can be seen in Figure 4, the initial stage of this research was carried out by collecting real data, which in this case is community mobility data during the COVID-19 pandemic. Furthermore, pre-processing is carried out on the data obtained, namely the process of normalizing the value of the community mobility index and also dividing the data set into training data and test data. The next stage is the design and implementation of the proposed model, namely GAOptimized Multivariate CNN-LSTM for Multi-channel Mobility Prediction. The proposed model is then tested using training data until the structure configuration that provides the best performance is found. After that, the performance of the proposed model was tested compared with the CNN and LSTM models used independently and the CNN-LSTM ensemble model whose parameters were not optimized. Based on the test results, then an evaluation of the performance comparison between the four models was carried out to confirm the performance of the GA-Optimized Multivariate CNN-LSTM for Multi-channel Mobility Prediction model. The final stage of this methodology is performance analysis and drawing conclusions based on the overall evaluation results and findings obtained. 


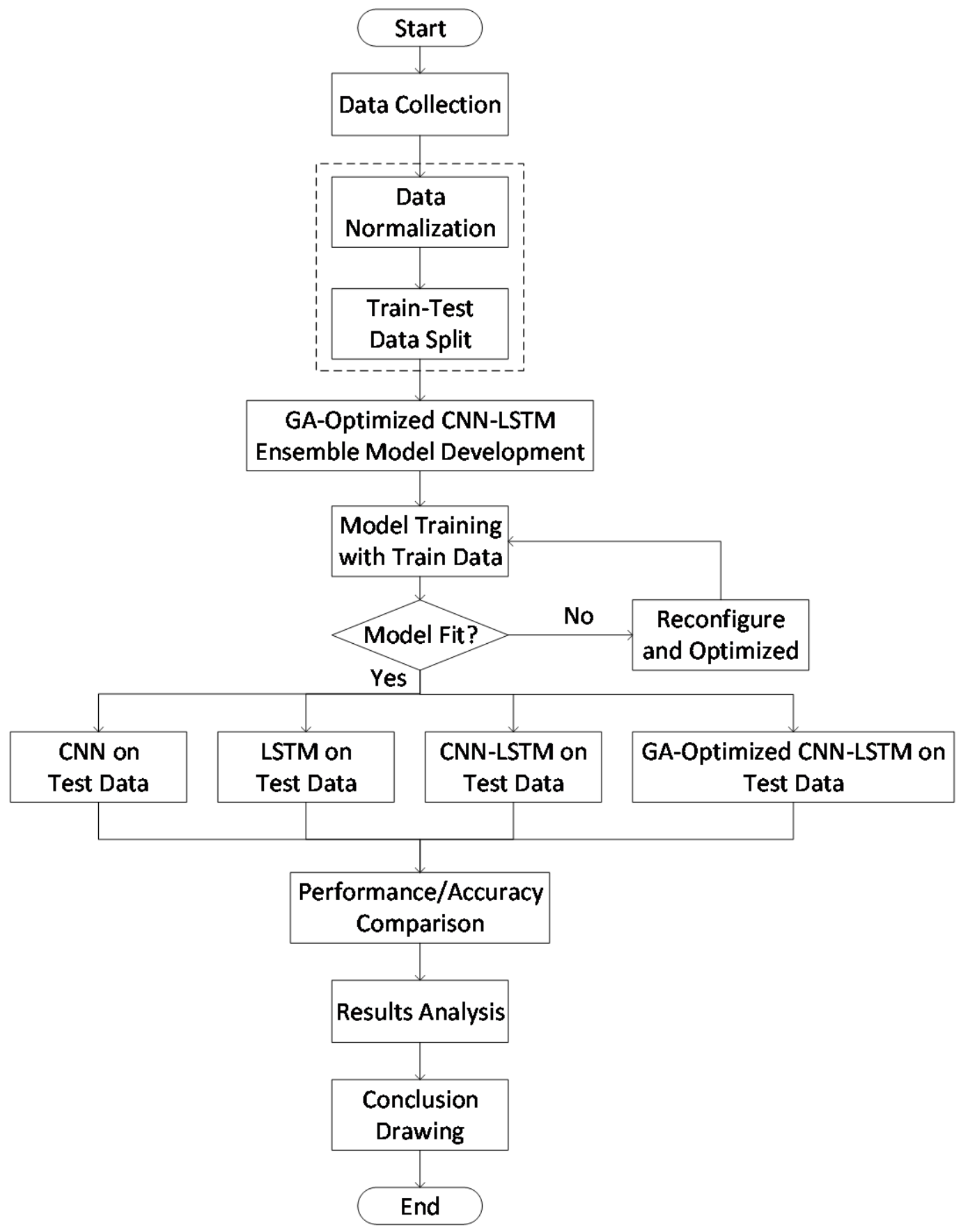

Figure 4. Constructive research methodology applied in the study.

\section{3-2-Description of Proposed Model}

When dealing with real-world variables, the value of one variable is often reliant on the past values of other variables as well. Factors such as income, interest rates, and investment expenditures, for example, may all have an impact on a household's spending costs. If all of these variables are related to consumer spending, it is logical to take their conditions into account when predicting consumer expenditure. In other terms, with $x_{1, t}, x_{2, t}, \ldots, x_{k, t}$ representing the associated variables, the prediction of $x_{1, t+h}$ at the end of time t may be expressed as follows:

$$
\hat{x}_{1, t+h}=f_{1}\left(x_{1, t}, x_{2, t}, \ldots, x_{k, t}, x_{1, t-1}, x_{2, t-1}, \ldots, x_{k, t-1}, x_{1, t-2}, x_{2, t-2}, \ldots, x_{k, t-2}, \ldots\right)
$$

Similarly, a second component prediction may be based on the system's past data. This equation may represent a $k^{t h}$ variable projection more broadly:

$$
\hat{x}_{k, t+h}=f_{k}\left(x_{1, t}, \ldots, x_{k, t}, x_{1, t-1}, \ldots, x_{k, t-1}, \ldots\right)
$$

A multivariate time-series has multiple time-dependent variables. Each variable is dependent on its previous value and other variables. Equation 2 represents the prediction of $\hat{x}_{k, t+h}$ as a function of a multiple time-series $x_{k, t}, k=$ 
$1, \ldots, k ; t=1, \ldots, t$. In univariate time-series analysis, finding suitable functions $f_{1}, \ldots, f_{q}$, i.e., the $q$-built functions are one of the most common primary goals. Nevertheless, finding out how variables interact is often a problem. For example, in a stock exchange setting with many markets, whether globally or locally, one may be interested in the effects of market interaction.

Accordingly, as stated before, this research focuses on people movement data in various areas during the COVID-19 epidemic since they are mostly multivariate time-series. Consequently, the effort in this research is focused on developing a trustworthy model capable of forecasting human movement trends in order to aid in the suppression of COVID-19 infection transmission. In this instance, CNN and LSTM may be used to construct deep learning models that can investigate complicated and hidden patterns in vast and varied data stacks, including time-series data, as previously described. Using the advantages of the two models, a time-series data forecasting model is created by combining CNN and LSTM, as well as including a multivariate time-series analysis method into the model to achieve the objectives of this study as presented in the Introduction, namely, to be able to predict patterns of human mobility in different localization during the COVID-19 pandemic.

The CNN-LSTM ensemble model would vary from commonly used time-series data forecasting methods, which typically use a univariate analytic strategy. The major distinction is that the CNN-LSTM model will make predictions based on correlation information between mobility channels as time-series. This is consistent with earlier research, which found that a collection of time-series data on the same topic has a tendency to have a connection and affect one another. Thus, while predicting future circumstances, information regarding the connection between series should be utilized.

As a result, the suggested and assessed model in this research is an ensemble of CNN and LSTM whose parameters will be further improved using GA to improve its performance. The multi-channel mobility data will be reshaped in this suggested architecture to match the input data structure that can be processed by the CNN structure and subsequently the LSTM. The GA-Optimized Multivariate CNN-LSTM model, named the GA CNN-LSTM in this paper, is made up of two main layers: the CNN layer, which is responsible for extracting the main features from the processed time-series data, and the LSTM layer, which is responsible for calculating the final prediction result and includes an optimization process to find the best parameter settings.

The GA CNN-LSTM model's structural diagram is shown in Figure 5, with CNN and LSTM as the main components, as well as an input layer, a 1-dimensional convolution layer (1D convolutional), a pooling layer, an LSTM hidden layer, and layer of fully connected hidden units, which will issue the prediction's final result. Accordingly, the size of kernel filters in the CNN layer and the number of hidden units in the LSTM layer are both optimized using GA in this architecture.

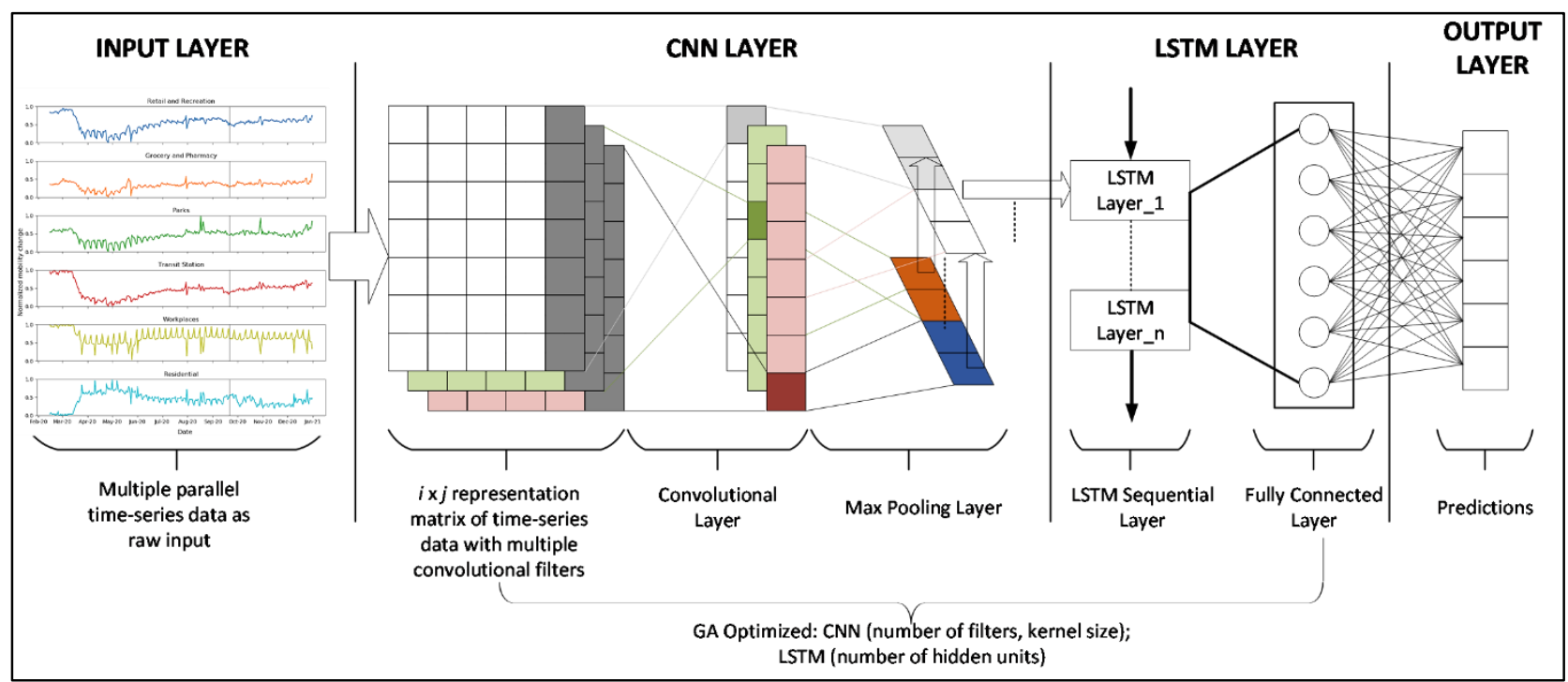

Figure 5. Structure of proposed GA-Optimized Multivariate CNN-LSTM (GA CNN-LSTM) for prediction of multi-channel mobility data that origins from six different area of activities.

As with other deep learning models, the GA CNN-LSTM model also has two main stages, namely the training or learning stage and the stage of utilizing trained models to perform predictive tasks. In the GA CNN-LSTM model, the proposed optimization process for the parameters is carried out at the learning stage. The sequence of the learning process is given as follows:

- The GA CNN-LSTM training stage starts with the input of training data. We now need to initialize each CNN and LSTM layer's settings, including weight and bias (if applicable). Then the process continues at the CNN layer, where the input data successively goes through the convolution and pooling layers, followed by the feature extraction procedure. The CNN layer extracts feature from the input time-series data. 
- The CNN layer's output value enters the LSTM layer. A multi-channel mobility series is predicted using the LSTM layer's output, which is then used as input by the complete connection layer to create the final predicted value. The prediction training procedure is now complete, and the outcomes are evaluated, with the error of the prediction results computed.

- The assessment findings are used to determine whether the training stopping criteria are fulfilled. The training ends when the prediction error value falls below a threshold, or the number of training cycles (epochs) is achieved.

- If not, the computed error is utilized to modify the weights and bias values at each layer (back propagation error), and the training procedure is repeated. To improve accuracy, the GA will increase the number of kernel filters in the CNN layer and the number of hidden units in the LSTM layer. If any of the stop criteria for training are satisfied, the training is finished, and the GA CNN-LSTM network configuration is stored.

After the learning process accompanied by the optimization process is complete, and the GA CNN-LSTM model has the ability to predict human movement with the expected degree of error, then the model can then be used to predict multi-channel mobility data. As a starting point of the prediction process, the test data used for prediction or testing data input is entered into the stored GA CNN-LSTM model, and the output value (prediction result) is then obtained as the final output of the CNN-LSTM training and prediction process. Following that, the degree of accuracy will be determined by calculating the Root Mean Square Error (RMSE) value to determine the amount of difference between the actual value and the predicted value.

\section{4- Experiment Settings}

This section describes the environmental settings used in the test to evaluate the performance of the proposed model that includes information about the data, architecture and configuration of the GA CNN-LSTM model as well as the evaluation process used to assess the performance of the tested model.

\section{4-1-Mobility Data}

As previously stated, human mobility has a significant impact on the transmission of COVID-19 infection. This is a frequent trend in situations where a disease has spread to the point of becoming a pandemic. As a result, the major emphasis of this work is human movement prediction, with the aim that the capacity to forecast patterns of community mobility may aid in anticipating the spread of illness. Accordingly, the data used in this study is data on human movement in an area which is collected and published by Google.

The Google Community Mobility Reports highlight mobility patterns by area and across various types of locations. Reports show changes in two ways for each category in a region: 1) a headline number that compares mobility for the report date to the baseline day, which is calculated for the report date (unless there are gaps) and reported as a positive or negative percentage; and 2) a trend graph that represents percent changes in the 6 weeks leading up to the report date.

As a result, the data indicates how visitors to (or time spent in) classified areas change over time in comparison to Google baseline days. A baseline day is the median value from the 5-week period from January 3 to February 6 , 2020 and reflects a normal value for that day of the week. As a result, the baseline days do not change. Google Community Mobility Reports are publicly available and may be seen at https:/www.google.com/covid19/mobility/.

The data in this research is restricted to Indonesian territory and covers the period from February 15 to December 31, 2020. The selection of data from Indonesia is based on the fact that Indonesia is one of Asia's most infected regions. Furthermore, Indonesia's vast population and extensive geographic coverage make it critical to be able to forecast and manage human movement. The first 120 days of data downloaded from Google Community Mobility Reports were utilized for training, while the following 102 days, from September 21 to December 31, 2020, were used for testing. The data utilized consists of six types of movement: retail and recreation, grocery and pharmacy, parks, transit stations, workplaces, and residential movements. Figure 6 shows the data plot, with the vertical line on the graph indicating the border region for training and test data. In this scenario, the test data is utilized as a reference for evaluating the performance of the assessed prediction model, while the training data is used for the learning process.

In addition to separating the data into training and test sets, the Google Community Mobility Reports data undergoes a normalization procedure, which involves mapping the index value of human movement to a range of 0.0 to 1.0 . This is done in order to improve the learning process as well as the prediction model's performance. Table 1 shows some of the variables from the Google Community Mobility Reports data that were utilized, as well as the outcomes of normalization. Meanwhile, the following mathematical formula is used to carry out the normalizing process:

$$
z_{i}=\frac{\left(x_{i}-(\min (x))\right.}{(\max (x)-\min (x))}
$$

where, $z_{i}$ is the $i^{\text {th }}$ normalized value in the dataset, $x_{i}$ is the $i^{\text {th }}$ value in the dataset, $\min (x)$ is the minimum value in the dataset, and $\max (x)$ is the maximum value in the dataset. 
Table 1. Snapshots of daily mobility report data in the Indonesia region. The Act notation represent the actual mobility index while the Norm notation represent the normalized index calculated by Equation 3.

\begin{tabular}{|c|c|c|c|c|c|c|c|c|c|c|c|c|}
\hline \multirow{3}{*}{ Date } & \multicolumn{12}{|c|}{ Mobility Data } \\
\hline & \multicolumn{2}{|c|}{ Retail and Recreation } & \multicolumn{2}{|c|}{ Grocery and Pharmacy } & \multicolumn{2}{|c|}{ Parks } & \multicolumn{2}{|c|}{ Transit Station } & \multicolumn{2}{|c|}{ Workplaces } & \multicolumn{2}{|c|}{ Residential } \\
\hline & Act & Norm & Act & Norm & Act & Norm & Act & Norm & Act & Norm & Act & Norm \\
\hline $15 / 02 / 2020$ & -2.00 & 0.84 & -2.00 & 0.36 & -8.00 & 0.54 & 1.00 & 0.96 & 5.00 & 0.99 & 1.00 & 0.04 \\
\hline $16 / 02 / 2020$ & -3.00 & 0.82 & -3.00 & 0.35 & -7.00 & 0.55 & -4.00 & 0.89 & 2.00 & 0.95 & 2.00 & 0.08 \\
\hline $17 / 02 / 2020$ & -3.00 & 0.82 & -4.00 & 0.34 & -7.00 & 0.55 & -1.00 & 0.93 & 3.00 & 0.96 & 1.00 & 0.04 \\
\hline $18 / 02 / 2020$ & -3.00 & 0.82 & -2.00 & 0.36 & -4.00 & 0.59 & 1.00 & 0.96 & 2.00 & 0.95 & 1.00 & 0.04 \\
\hline $19 / 02 / 2020$ & -3.00 & 0.82 & -4.00 & 0.34 & -3.00 & 0.6 & 0.00 & 0.94 & 1.00 & 0.94 & 1.00 & 0.04 \\
\hline $20 / 02 / 2020$ & -2.00 & 0.84 & -1.00 & 0.37 & -1.00 & 0.62 & 4.00 & 1.00 & 1.00 & 0.94 & 1.00 & 0.04 \\
\hline $21 / 02 / 2020$ & -2.00 & 0.84 & -3.00 & 0.35 & -5.00 & 0.58 & 2.00 & 0.97 & 5.00 & 0.99 & 1.00 & 0.04 \\
\hline $22 / 02 / 2020$ & 0.00 & 0.87 & 0.00 & 0.38 & -5.00 & 0.58 & 1.00 & 0.96 & 5.00 & 0.99 & 0.00 & 0.00 \\
\hline $23 / 02 / 2020$ & -2.00 & 0.84 & -1.00 & 0.37 & -5.00 & 0.58 & -4.00 & 0.89 & 3.00 & 0.96 & 1.00 & 0.04 \\
\hline $24 / 02 / 2020$ & -2.00 & 0.84 & -3.00 & 0.35 & -4.00 & 0.59 & -1.00 & 0.93 & 4.00 & 0.97 & 1.00 & 0.04 \\
\hline
\end{tabular}
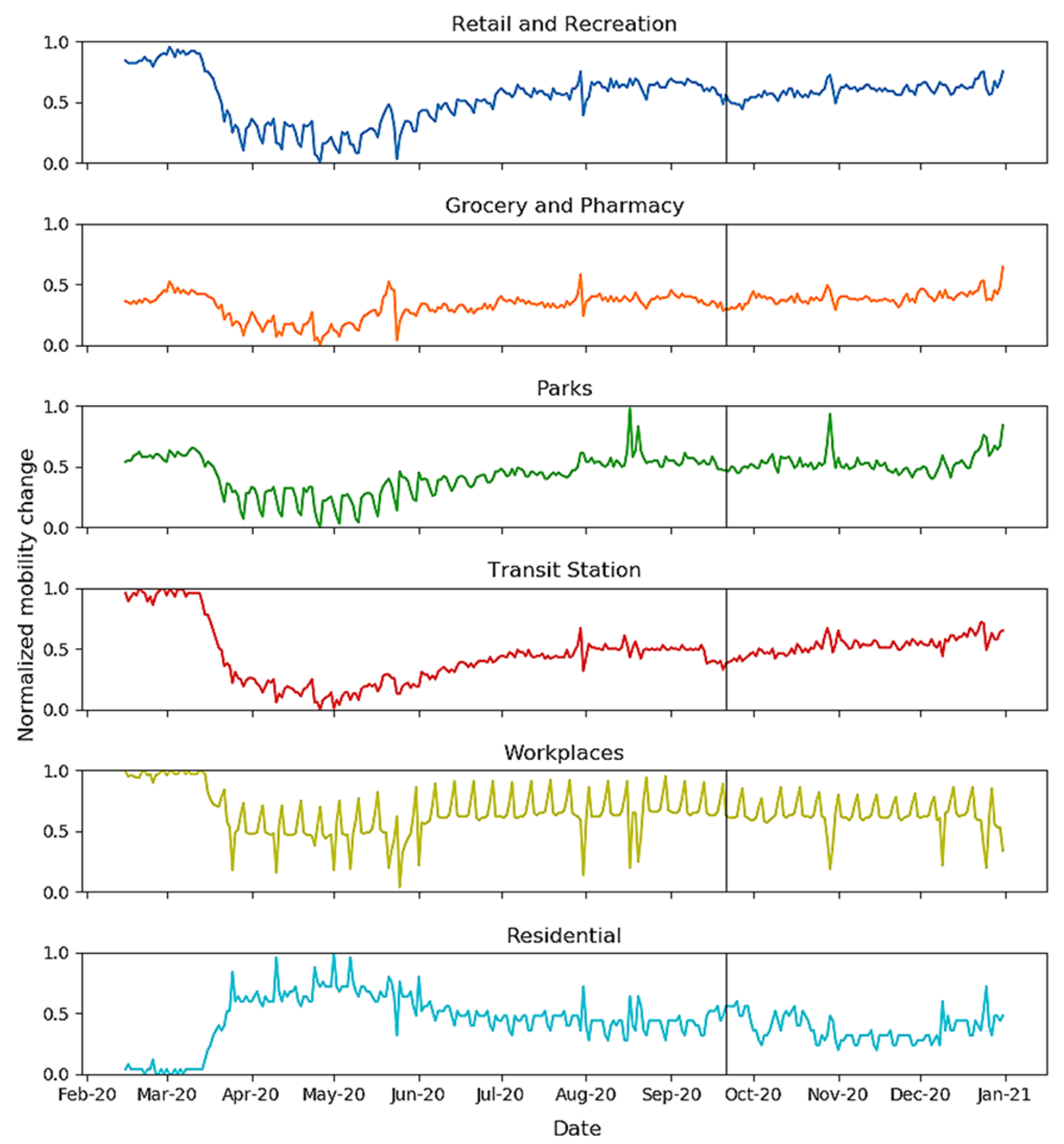

Figure 6. Plots of The Google Community Mobility Reports ranging from February 15 to December 31, 2020, in Indonesia. The first 120 days is used as training set while the last 102 days is used as test set. The training and testing part is separated by the vertical line. 
Brief observations of the movement graph plotted in Figure 6 reveal a shift in the normal movement pattern, particularly in the movement graph in the Workplaces and Residential regions, which began to spread considerably in Indonesia in March 2020. A reduction in the daily movement index in the work area and an increase in the movement index in the residential area were observed. Furthermore, the pattern appears to be repeated weekly and remains quite steady until the conclusion of the year. As a result, it's possible to infer that there's a significant link between workplace and residential mobility patterns, which must be considered when forecasting future movement indices.

\section{4-2-Architecture of GA CNN-LSTM for Multi-channel Mobility Prediction}

The structure utilized in both the CNN, LSTM, CNN-LSTM, and GA CNN-LSTM models is a version of the simplest to guarantee that the use of GA for parameter optimization in the CNN-LSTM ensemble offers enhanced performance and to evaluate the performance of the four models equitably. In this example, the CNN model only uses one convolution layer, the LSTM model only uses one hidden layer, the CNN-LSTM ensemble model uses one convolution layer and one hidden layer each, and the GA CNN-LSTM utilizes the same structure as the CNN-LSTM ensemble.

Table 2. Parameters configuration for the proposed GA CNN-LSTM model. The convolutional layer filters and number of LSTM layer hidden unit values are initially set to 64 and 100 and will be optimized by GA throughout learning process.

\begin{tabular}{lcc}
\hline \multicolumn{1}{c}{ Parameters } & Values & Layer \\
\hline Convolutional layer filters & $\mathbf{6 4}$ (initial) & CNN \\
Convolutional layer kernel_size & 2 & CNN \\
Convolutional layer activation function & Relu & CNN \\
Convolutional layer padding & Valid & CNN \\
Pooling layer pool_size & 2 & CNN \\
Number of LSTM layer hidden unit & $\mathbf{1 0 0}$ (initial) & LSTM \\
LSTM hidden layer activation function & Relu & LSTM \\
Time step & 7 & LSTM \\
Optimizer & Adam & Model Fitting \\
Loss function & MAE & Model Fitting \\
Epochs & 1000 & Model Fitting \\
\hline
\end{tabular}

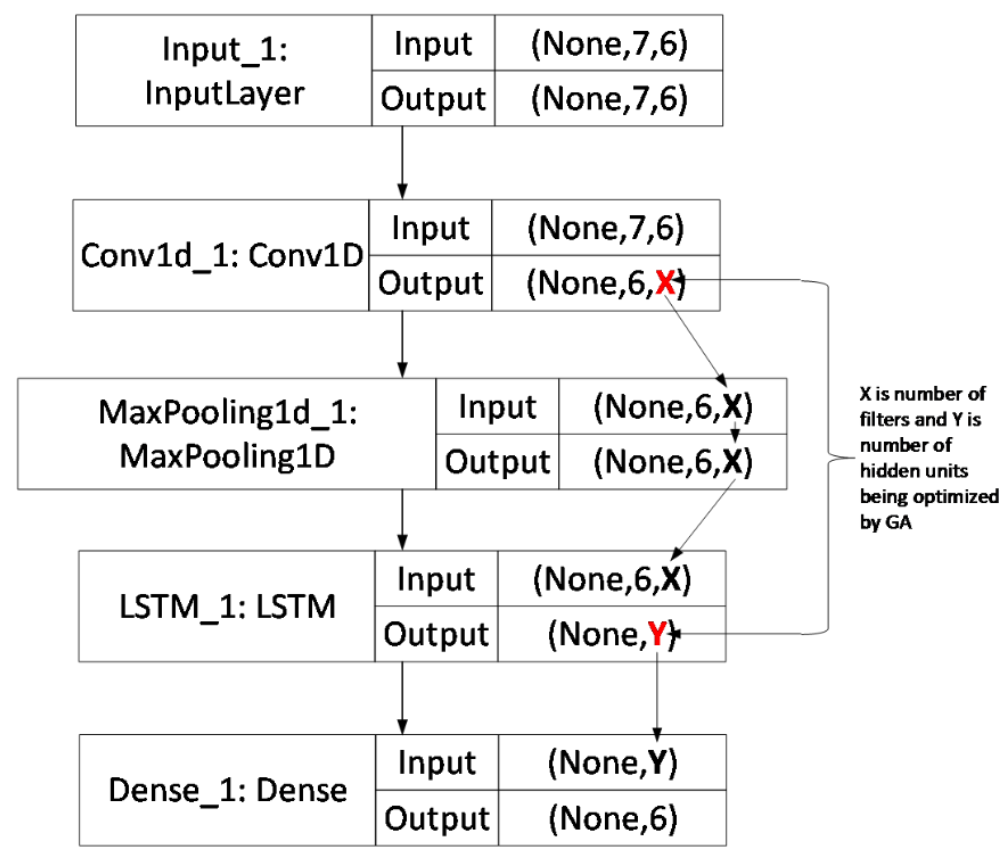

Figure 7. Architecture of the proposed GA CNN-LSTM model for multi-channel mobility prediction with 6 features and 7time steps for the prediction process.

Table 2 also shows the GA CNN-LSTM model's initial architectural setup (prior to GA's optimization procedure). It can be deduced that the basic model is designed as follows based on the CNN-LSTM ensemble network's parameter settings: the input training set data is a three-dimensional data vector (None, 7, 6), where the first number 7 represents the time step size and the other number 6 is the input dimensions of six movement indexes from six different area, which in this case are movement in the retail and recreation, grocery and pharmacy, parks, transit stations, workplaces, and residential area. 
The data is initially sent through a one-dimensional convolution layer, which extracts additional features and generates a three-dimensional output vector (None, 7, 64), with 64 being the initial size of the convolution layer filters. The vector is then joined to the pooling layer and transformed into a three-dimensional output vector (None, 7, 64). The output vector is then sent to the LSTM hidden layer with shape (None, 100) for training; 100 is the initial number of hidden units in the LSTM hidden layer; after training, the output data is sent to the LSTM dense layer to produce output values, i.e., the prediction.

Parameter settings, as shown in Table 2, refers to the structure and configuration of parameter values used by [29] in their CNN-LSTM ensemble model for stock prices prediction in the Shanghai Composite Index. The main adjustments made in our proposed model are the initial value of the convolutional filters on CNN and the number of hidden units on the LSTM. In their conducted research, Lu et al. used 32 convolutional filters and 64 hidden units to predict univariate stock price movements. The change in the number of convolutional filters and hidden units in the model proposed in this study is due to the multivariate character of the mobility time-series data, so that more convolutional filters and hidden units are needed to be able to carry out the calculation process. Utilization of the parameter values configuration as used by [29] in their research, is based on the results of the research trials which indicate that the CNN-LSTM ensemble architecture built has good performance.

In this proposed model setting, the initial size of the convolution layer filters in the CNN, which is set to 64, and the initial number of hidden units in the LSTM, which is set to 100, is being optimized throughout the training process by GA until the best result i.e., the lowest error is achieved and therefore would change alternately. As a result, Figure 7 illustrates the suggested CNN-LSTM model's fundamental structure, where $\mathbf{X}$ represents the optimized size the convolution layer filters in CNN whilst $\mathbf{Y}$ represents the optimized number of hidden units in the LSTM.

\section{4-3-Model Evaluation}

Calculating the error value of the predictions made at each point in time is used to evaluate the performance of the proposed model and other models as a comparison. In addition, the statistical features of the prediction results obtained with the original data are compared in the assessment process. The descriptive statistical value between the original data and the projected outcomes was calculated in a comparative study of statistical features.

The purpose of evaluating the error value of the prediction is to be able to analyze the performance of the prediction model in terms of accuracy. The root mean square error (RMSE) of each projected mobility data group is utilized in this scenario. For each assessed model, namely CNN, LSTM, CNN-LSTM, and GA CNN-LSTM, RMSE values were computed. The following mathematical formula is used to determine the RMSE value:

$$
R M S E=\sqrt{\frac{1}{n} \sum_{i=1}^{n}\left(\widehat{y}_{l}-y_{i}\right)^{2}}
$$

where $\widehat{y}_{l}$ is the predicted value at a particular time-point $i$ and $y_{i}$ is the actual value. Since the difference between the forecast and the original value is less, a lower RSME value means higher prediction accuracy.

Additionally, descriptive statistics give easy-to-understand descriptions of the sample and the observations made. These summaries might be quantitative, such as summary statistics, or visual, such as simple graphics. These summaries may serve as the foundation for an initial description of the data as part of a larger statistical study, or they may be adequate for a specific research on their own. Therefore, two groups of data sets with similar descriptive statistical values can be considered to have similar characteristics and in the case of predictive data, it can be assumed that the prediction results have a high degree of similarity to the original value.

\section{5- Results and Discussion}

As previously stated, this research is based on data on people's mobility in Indonesia across six different areas: retail and leisure, supermarket and pharmacy, parks, transit stops, workplaces, and residential. The data utilized is based on 222 daily motions, with some of the data being used as training data and some being used as test data. The GA CNNLSTM model presented in this research was used to train and evaluate the prediction outcomes on four deep learning models: CNN, LSTM, CNN and LSTM ensembles, and the GA CNN-LSTM model.

Figure 8 shows a visualization of each model's predictions for people mobility in workplaces and residential regions compared to the original data. The solid line in the image shows the original value plot, while the dotted line indicates each model's projected plot. Furthermore, the same graph shows the size of the forecast inaccuracy with comparison to the original value, which is represented by the yellow region. In-depth analysis of the graph reveals that the performance of the CNN, LSTM, and CNN-LSTM ensemble models in forecasting people's movements in businesses and residential areas is almost identical. This condition is also present in the predictions for the other four types of community mobility, namely retail and recreation, supermarket and pharmacy, parks, and transit stops. Table 3, which shows information on the RMSE value of each model in each set of community mobility areas, confirms this finding. 
As a result, it was discovered early on that the CNN and LSTM models had a significant capacity to anticipate people's movement in multi-channels. Furthermore, combining the two models into an ensemble model may enhance prediction outcomes at particular periods in time. The CNN-LSTM ensemble model, on the other hand, cannot be overall claimed to outperform the CNN and LSTM models consistently. This can be seen in Figure 7, where the yellow region on the comparison graph of each model's prediction results shows this. In general, the yellow area in the movement forecast of the CNN, LSTM, and CNN-LSTM ensemble models tends to be almost identical. In the workplace and in residential settings, there is a sense of community.
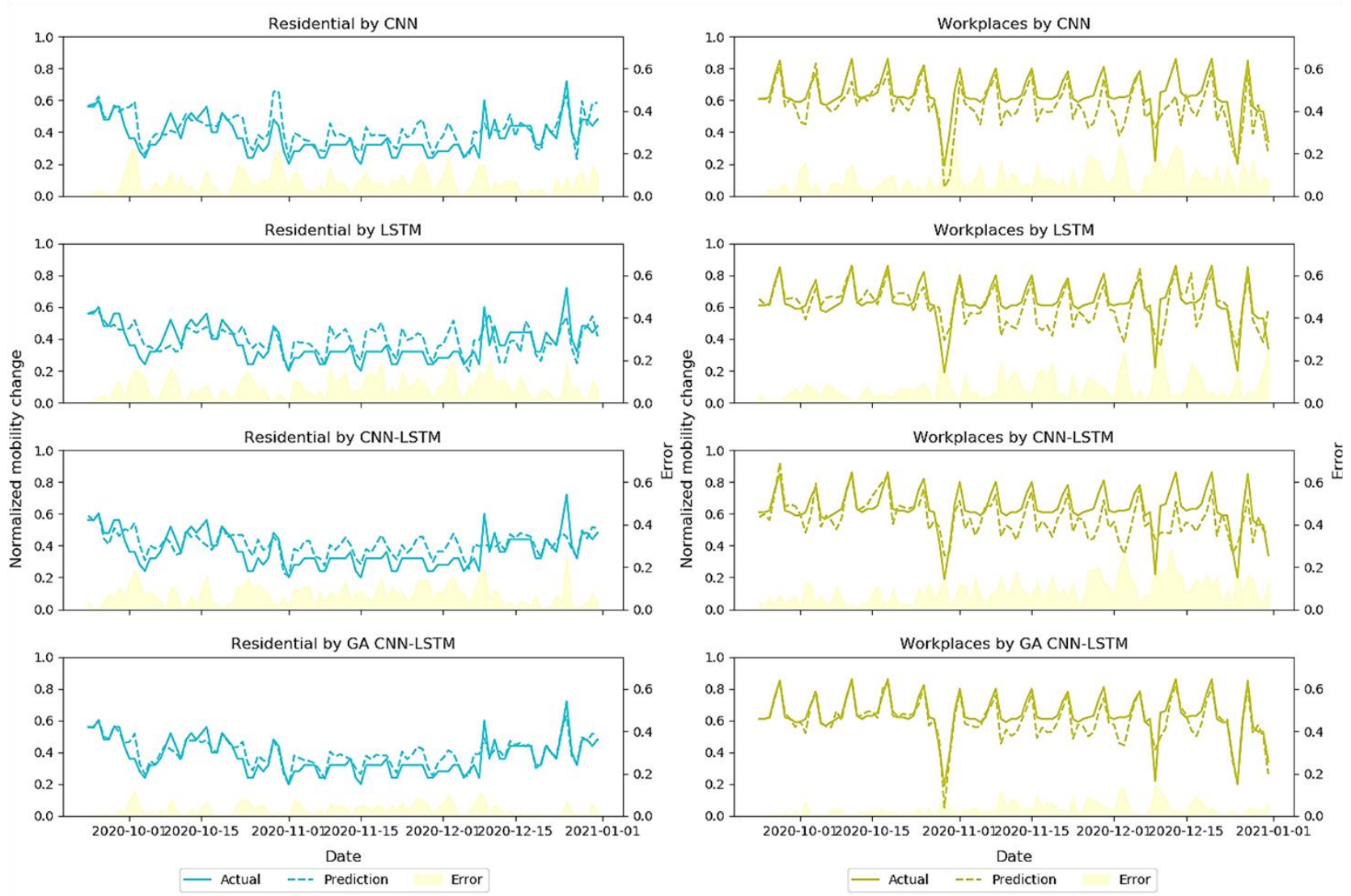

Figure 8. Plot of comparison between actual and prediction of mobility index for workplaces and residential area in Indonesia by CNN, LSTM, ensemble of CNN-LSTM and GA CNN-LSTM.

Table 3. Comparison of RMSE for each mobility area produced by CNN, LSTM, ensemble of CNN-LSTM and GA CNNLSTM when predicting mobility index in Indonesia.

\begin{tabular}{ccccc}
\hline \multirow{2}{*}{ Mobility Data } & \multicolumn{4}{c}{ Prediction RMSE } \\
\cline { 2 - 5 } & CNN & LSTM & CNN-LSTM & GA CNN-LSTM \\
\hline Retail and Recreation & 0.0726 & 0.0590 & 0.0590 & 0.0353 \\
Grocery and Pharmacy & 0.0695 & 0.0429 & 0.0732 & 0.0299 \\
Parks & 0.0538 & 0.0730 & 0.0689 & 0.0412 \\
Transit Station & 0.0893 & 0.0584 & 0.0918 & 0.0485 \\
Workplaces & 0.1035 & 0.0945 & 0.1087 & 0.0625 \\
Residential & 0.0827 & 0.0881 & 0.0848 & 0.0555 \\
\hline
\end{tabular}

However, the comparison plot of the GA CNN-LSTM model's prediction outcomes to the original value reveals a substantial difference. Figure 8 clearly indicates that the yellow area in the GA CNN-LSTM model in the workplaces and residential regions, which reflects the magnitude of the prediction error value, seems to be lower than the other three models. As can be seen from the RMSE values in Table 3, this finding is equally constant in the other four domains of community mobility. The RMSE values produced by the GA CNN-LSTM model is consistently lower in the six areas of community mobility than the other three models, as shown in Table 3.

If the root mean squared error (RMSE) values in Table 3 are examined in greater depth, it is frequently noted that the largest predictive salience value occurs in time-series data collected from workplaces and residential areas. This condition is present in all of the models that were evaluated in this study. When we look at the data on people's mobility in the six region categories depicted in Figure 6, we can see that individuals who likely to have pretty regular movement 
patterns are those who work in offices and live-in residential areas. Figure 6 further demonstrates that there is little change in movement in the other four sections of the graph. This situation offers a clear evidence that people's travels, particularly in Indonesia, during the COVID-19 epidemic occur mostly in the vicinity of their residence or place of employment. At the same time, people's moves in other regions tend to be less volatile, which results in a low degree of prediction error.

The pattern of community mobility in the region of living and employment is also in the opposite direction of the previous pattern. Increasing activity in the residential area leads to a decrease in movement in the work area, as seen by Figure 6, whereas the opposite is true for the work area. People spend more time and carry out their activities in their neighborhoods and at work during the COVID-19 epidemic, but not in other places, according to these findings. Both locations have higher than average prediction error values, which shows that the more volatile time-series data will result in lower performance of the model that was used to forecast the time-series values in both areas.

Accordingly, Figure 9 compares the prediction results calculated by the CNN-LSTM ensemble model to the GA CNN-LSTM model, confirming previous findings that the GA CNN-LSTM model is consistently able to produce predictions of community movement indexes that are closer to the movement of the original values in all six areas. The figure of the prediction error rate supports the prior results, with Error-1 representing the prediction error produced by the CNN-LSTM ensemble model and Error-2 representing the prediction error created by the GA CNN-LSTM model. In this instance, the area of Error-2 is always lower than the area of Error-1 in the six series, as can be seen in Figure 9.

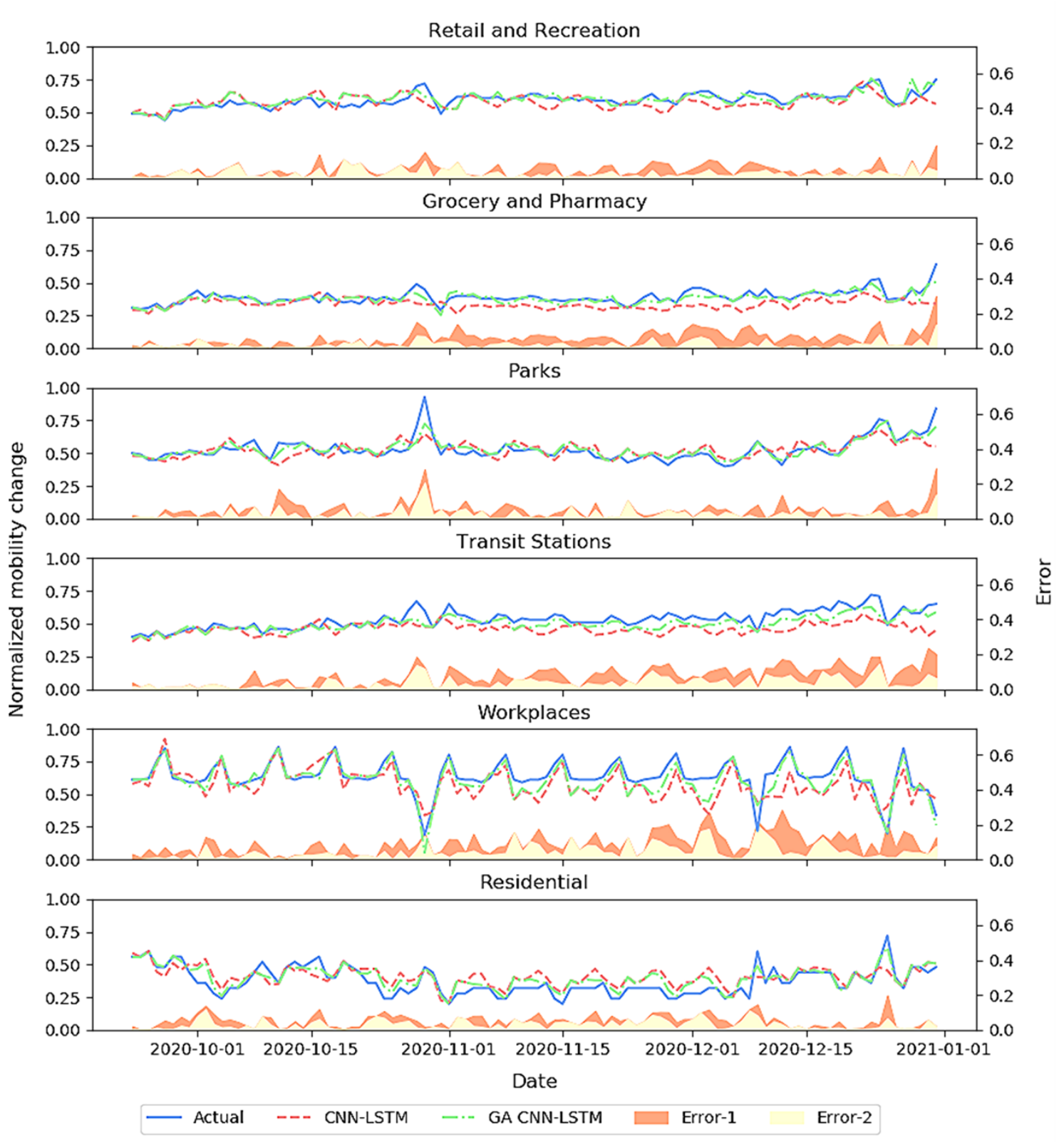

Figure 9. Comparison of actual values and prediction in six mobility area by ensemble of CNN-LSTM and GA CNN-LSTM. The Error-1 represents error in prediction produced by ensemble of CNN-LSTM while Error-2 represents error in prediction produced by GA CNN-LSTM. 
Furthermore, as shown in Figure 10, the comparison graph of each model's RMSE values in each region of community movement indicates that the CNN, LSTM, and CNN-LSTM ensemble models have inconsistent performance and change from one set of movement areas to the next. Meanwhile, in each of the six movement regions, the GA CNN-LSTM model consistently produces the lowest RMSE value.

Based on the facts presented and the outcomes of the tests conducted, it can be concluded that putting up an ensemble by simply merging multiple models that have been shown to perform well, particularly in forecasting time-series data, does not guarantee excellent results. Better on a regular basis However, in order to accomplish the primary objective of the ensemble building, which is to enhance performance, particularly the accuracy of prediction outcomes, an optimization procedure on the parameters that exist in these models is required. The size of the kernel filters at the CNN layer, as well as the number of hidden units at the LSTM layer, are the parameters optimized by GA in this experiment, as previously stated. The most optimum kernel filter size value was 96 from an initial value of 64, and the number of hidden units was 120 from an initial value of 100 at the conclusion of the training procedure.

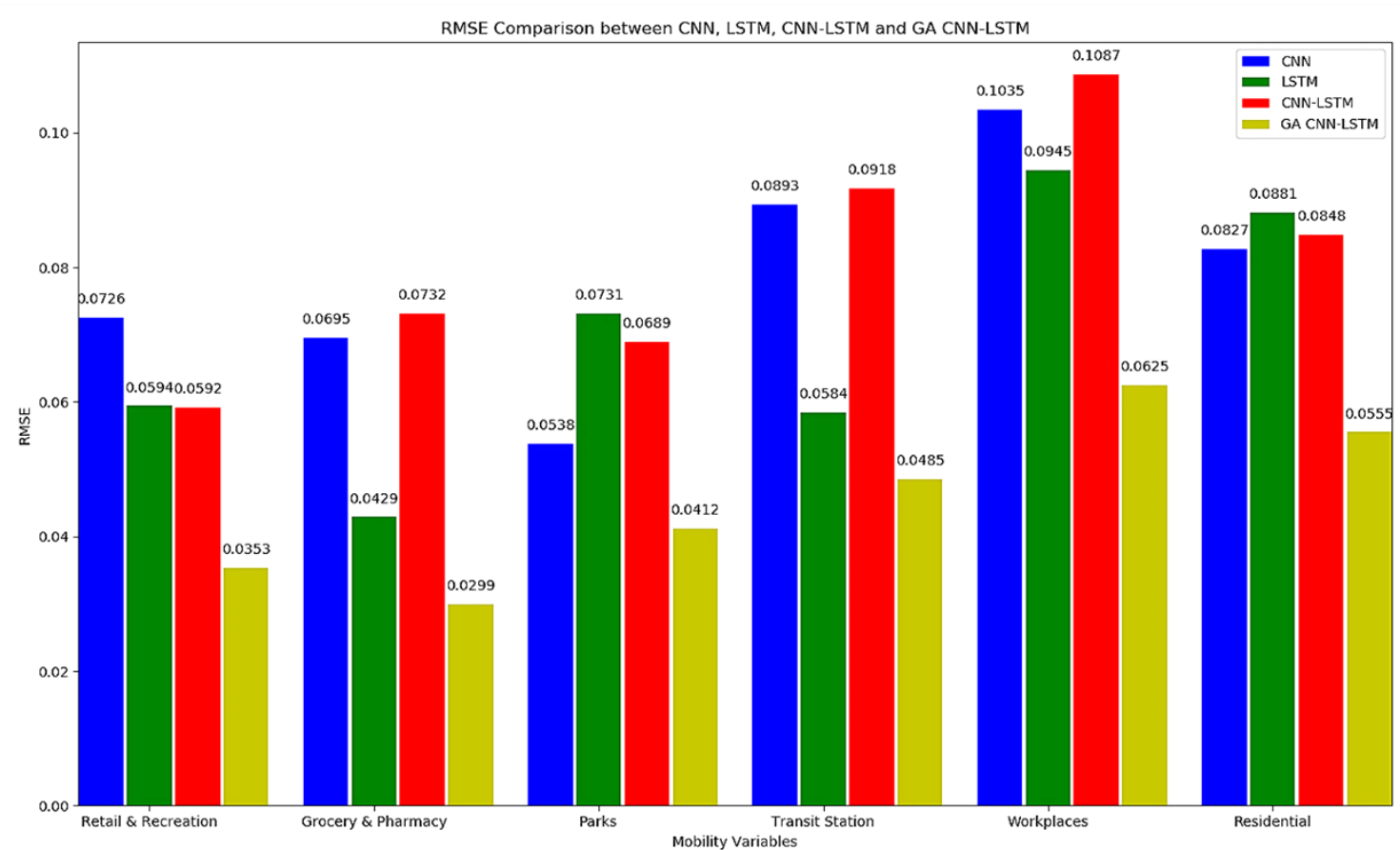

Figure 10. RMSE produced by each evaluated model for each mobility area.

Table 4 provides information on the descriptive statistical value of the predicted results compared to the original value, which complements the analysis of the prediction results measured by the RMSE quantity to assess the performance of the GA CNN-LSTM model in terms of prediction accuracy. In general, the descriptive statistical values of the two data sets are not significantly different, indicating that the GA CNN-LSTM model's projected data set has statistical features comparable to the original data. This demonstrates that the GA CNN-LSTM model is capable of generating high-accuracy predictions while still having features that are comparable to the original data.

Table 4. Descriptive statistics of actual values compared to predictions made by GA CNN-LSTM.

\begin{tabular}{|c|c|c|c|c|c|c|c|c|c|c|c|c|}
\hline \multirow{3}{*}{$\begin{array}{l}\text { Descriptive } \\
\text { Statistics }\end{array}$} & \multicolumn{12}{|c|}{ Mobility Data } \\
\hline & \multicolumn{2}{|c|}{$\begin{array}{l}\text { Retail and } \\
\text { Recreation }\end{array}$} & \multicolumn{2}{|c|}{$\begin{array}{l}\text { Grocery and } \\
\text { Pharmacy }\end{array}$} & \multicolumn{2}{|c|}{ Parks } & \multicolumn{2}{|c|}{ Transit Station } & \multicolumn{2}{|c|}{ Workplaces } & \multicolumn{2}{|c|}{ Residential } \\
\hline & Act & Pred & Act & Pred & Act & Pred & Act & Pred & Act & Pred & Act & Pred \\
\hline Mean & 0.5952 & 0.6000 & 0.3906 & 0.3797 & 0.5299 & 0.5329 & 0.5322 & 0.4997 & 0.6357 & 0.6054 & 0.3744 & 0.3995 \\
\hline Median & 0.5900 & 0.5988 & 0.3800 & 0.3823 & 0.5100 & 0.5299 & 0.5300 & 0.4904 & 0.6200 & 0.6011 & 0.3600 & 0.3918 \\
\hline Standard Deviation & 0.0564 & 0.0536 & 0.0505 & 0.0426 & 0.0848 & 0.0633 & 0.0689 & 0.0537 & 0.1240 & 0.1312 & 0.1055 & 0.0853 \\
\hline Sample Variance & 0.0032 & 0.0029 & 0.0025 & 0.0018 & 0.0072 & 0.0040 & 0.0048 & 0.0029 & 0.0154 & 0.0172 & 0.0111 & 0.0073 \\
\hline Kurtosis & 0.9368 & 1.4059 & 5.6540 & 1.3250 & 5.9676 & 1.7128 & -0.0642 & 0.0107 & 3.4630 & 3.0184 & 0.0054 & 0.0062 \\
\hline Skewness & 0.2256 & 0.1777 & 1.4897 & 0.2047 & 2.0121 & 1.1417 & 0.3526 & 0.2276 & -1.0851 & -0.9136 & 0.6608 & 0.1527 \\
\hline Range & 0.3100 & 0.3149 & 0.3500 & 0.2507 & 0.5300 & 0.3109 & 0.3200 & 0.2562 & 0.6700 & 0.7962 & 0.5200 & 0.4340 \\
\hline Minimum & 0.4400 & 0.4442 & 0.2900 & 0.2547 & 0.4000 & 0.4363 & 0.4000 & 0.3712 & 0.1900 & 0.0533 & 0.2000 & 0.1964 \\
\hline Maximum & 0.7500 & 0.7591 & 0.6400 & 0.5054 & 0.9300 & 0.7472 & 0.7200 & 0.6274 & 0.8600 & 0.8495 & 0.7200 & 0.6304 \\
\hline Conf. Level (95.0\%) & 0.0112 & 0.0106 & 0.0100 & 0.0085 & 0.0168 & 0.0126 & 0.0137 & 0.0107 & 0.0246 & 0.0260 & 0.0209 & 0.0169 \\
\hline
\end{tabular}


The suggested model has potential performance based on the graph plot of the prediction results compared to the original value, the error rate calculation in RMSE, and the comparison of descriptive statistical values between the projected results and the original values. In this regard, a comparison of the findings obtained in this study with those obtained in prior studies using similar data sets can undoubtedly assist to corroborate the performance of the suggested model. However, research on predicting the spread of COVID-19 infection has so far concentrated on projecting the amount of dissemination or the impact of lockdown measures on community mobility, rather than forecasting the level of mobility itself. For example, research by [39] revealed that combining multiple factors, including the degree of mobility, may yield an accurate estimate of the spread of COVID-19 infection, while study by [40] assessed the influence of lock down regulations on the level of community mobility. As a result, the evaluation of the acquired experimental results at this time cannot be compared to prior research since no studies with similar circumstances, such as the same dataset, settings, and issues to be solved, have been discovered.

Nevertheless, various results obtained from the trials carried out in this study indicate that the GA CNN-LSTM model has the ability to recognize patterns of community movement in six different areas. Furthermore, the GA CNN-LSTM model is also able to provide prediction results with the smallest error rate compared to the CNN, LSTM and CNNLSTM ensemble models. Therefore, this proposed model can be considered as an alternative technique for forecasting community movements during the COVID-19 pandemic, especially in Indonesia. Having the ability to predict trends in people's movements in various areas during this pandemic will be able to help policymakers or governments plan more effective movement restrictions to reduce the rate of spread of COVID-19 infections.

\section{6- Conclusion and Future Work}

This research presents an architecture consisting of an ensemble of two deep learning models frequently used for time-series data prediction, namely CNN and LSTM, whose structures have been changed to learn patterns of movement of multivariate time-series data and predict future values. Referring to the current state of the spread of the COVID-19 pandemic, the performance of the proposed model was tested using data on community mobility in the Indonesian region. The selection of mobility data as time-series test data is based on the fact that community mobility is one of the main factors in the spread of COVID-19 infection. This study also conducted a comparative analysis of the performance of the CNN-LSTM ensemble whose parameters were optimized using GA to determine the importance of tuning the ensemble model that was built to solve a problem, such as predicting time-series data. Based on the results of the experiments conducted, it was determined that the prediction model of people's movements during the COVID-19 epidemic, which was built using an ensemble of CNN and LSTM models optimized with GA, is capable of providing forecasts for multivariate time-series data with a high degree of precision. Additional to this, the evaluation of four different models (CNN, LSTMA, CNN-LSTM, and GA CNN-LSTM) revealed that simply coupling CNN and LSTM models directly without optimizing the existing parameters does not guarantee that predictions will be generated with the highest level of accuracy at every time point. Consequently, based on piece of evidence presented above, it can be stated that the GA CNN-LSTM model may be used to anticipate community migrations, particularly during the COVID19 epidemic. This can therefore be used as a reference to assist policymakers or the government in creating regulations to restrict people's movements in order to aid in the suppression of the spread of COVID-19 infection. Additionally, to facilitate further development of the study, it is intended to expand the number of parameters to be optimized in the GA CNN-LSTM architecture, such as the number of hidden layers, the type of activation function, and other parameters' setting.

\section{7- Declarations}

\section{7-1-Data Availability Statement}

Data available in a publicly accessible repository: The data presented in this study are openly available in Google COVID-19 Community Mobility Reports at https://www.google.com/covid19/mobility/.

\section{7-2- Funding}

This work is supported by the Perbanas Institute, Jakarta, Indonesia and the Ministry of Education, Culture, Research and Technology of the Republic of Indonesia.

\section{7-3-Acknowledgements}

The author wishes to express his gratitude to Perbanas Institute and the Ministry of Education, Culture, Research and Technology of the Republic of Indonesia for their support of this study.

\section{7-4- Conflicts of Interest}

The authors declare that there is no conflict of interests regarding the publication of this manuscript. In addition, the ethical issues, including plagiarism, informed consent, misconduct, data fabrication and/or falsification, double publication and/or submission, and redundancies have been completely observed by the authors. 


\section{8- References}

[1] Changruenngam, Suttikiat, Dominique J. Bicout, and Charin Modchang. "How the Individual Human Mobility Spatio-Temporally Shapes the Disease Transmission Dynamics.” Scientific Reports 10, no. 1 (July 9, 2020). doi:10.1038/s41598-020-68230-9.

[2] Gao, Daozhou. “How Does Dispersal Affect the Infection Size?” SIAM Journal on Applied Mathematics 80, no. 5 (January 2020): 2144-2169. doi:10.1137/19m130652x.

[3] Wang, Yi, Zhouchao Wei, and Jinde Cao. "Epidemic Dynamics of Influenza-Like Diseases Spreading in Complex Networks." Nonlinear Dynamics 101, no. 3 (August 2020): 1801-1820. doi:10.1007/s11071-020-05867-1.

[4] Wong, David W. S., and Yun Li. "Spreading of COVID-19: Density Matters.” Edited by Bing Xue. PLOS ONE 15, no. 12 (December 23, 2020): e0242398. doi:10.1371/journal.pone.0242398.

[5] Ribeiro, Haroldo V., Andre S. Sunahara, Jack Sutton, Matjaž Perc, and Quentin S. Hanley. "City Size and the Spreading of COVID-19 in Brazil." Edited by Luo-Luo Jiang. PLOS ONE 15, no. 9 (September 23, 2020): e0239699. doi:10.1371/journal.pone.0239699.

[6] Zhang, Jie, Baoheng Feng, Yina Wu, Pengpeng Xu, Ruimin Ke, and Ni Dong. "The Effect of Human Mobility and Control Measures on Traffic Safety during COVID-19 Pandemic." Edited by Feng Chen. PLOS ONE 16, no. 3 (March 8, 2021): e0243263. doi:10.1371/journal.pone.0243263.

[7] Fakir, Adnan M. S., and Tushar Bharati. "Pandemic Catch-22: The Role of Mobility Restrictions and Institutional Inequalities in Halting the Spread of COVID-19." Edited by Faisal Abbas. PLOS ONE 16, no. 6 (June 28, 2021): e0253348. doi:10.1371/journal.pone.0253348.

[8] Giles, John R., Elisabeth zu Erbach-Schoenberg, Andrew J. Tatem, Lauren Gardner, Ottar N. Bjørnstad, C. J. E. Metcalf, and Amy Wesolowski. "The Duration of Travel Impacts the Spatial Dynamics of Infectious Diseases." Proceedings of the National Academy of Sciences 117, no. 36 (August 24, 2020): 22572-22579. doi:10.1073/pnas.1922663117.

[9] Tao, Haiyan, Keli Wang, Li Zhuo, Xuliang Li, Qiuping Li, Yuan Liu, and Yong Xu. "A Comprehensive Framework for Studying Diffusion Patterns of Imported Dengue with Individual-Based Movement Data." International Journal of Geographical Information Science 34, no. 3 (November 18, 2019): 604-624. doi:10.1080/13658816.2019.1684497.

[10] Liu, Kun, Siqi Ai, Shuxuan Song, Guanghu Zhu, Fei Tian, Huan Li, Yuan Gao, et al. "Population Movement, City Closure in Wuhan, and Geographical Expansion of the COVID-19 Infection in China in January 2020." Clinical Infectious Diseases 71, no. 16 (April 17, 2020): 2045-2051. doi:10.1093/cid/ciaa422.

[11] Lai, Shengjie, Nick W. Ruktanonchai, Liangcai Zhou, Olivia Prosper, Wei Luo, Jessica R. Floyd, Amy Wesolowski, et al. "Effect of Non-Pharmaceutical Interventions to Contain COVID-19 in China." Nature 585, no. 7825 (May 4, 2020): 410-413. doi:10.1038/s41586-020-2293-x.

[12] Chang, Meng-Chun, Rebecca Kahn, Yu-An Li, Cheng-Sheng Lee, Caroline O. Buckee, and Hsiao-Han Chang. "Variation in Human Mobility and Its Impact on the Risk of Future COVID-19 Outbreaks in Taiwan.” BMC Public Health 21, no. 1 (January 27, 2021). doi:10.1186/s12889-021-10260-7.

[13] Sainath, Tara N., Brian Kingsbury, Abdel-rahman Mohamed, George E. Dahl, George Saon, Hagen Soltau, Tomas Beran, Aleksandr Y. Aravkin, and Bhuvana Ramabhadran. “Improvements to Deep Convolutional Neural Networks for LVCSR.” 2013 IEEE Workshop on Automatic Speech Recognition and Understanding (December 2013): 8614-8618. doi:10.1109/asru.2013.6707749.

[14] Ciresan, D., U. Meier, and J. Schmidhuber. "Multi-Column Deep Neural Networks for Image Classification." 2012 IEEE Conference on Computer Vision and Pattern Recognition (June 2012): 3642-3649. doi:10.1109/cvpr.2012.6248110.

[15] Brocardo, Marcelo Luiz, Issa Traore, Isaac Woungang, and Mohammad S. Obaidat. “Authorship Verification Using Deep Belief Network Systems." International Journal of Communication Systems 30, no. 12 (January 18, 2017): e3259. doi:10.1002/dac.3259.

[16] Silver, David, Aja Huang, Chris J. Maddison, Arthur Guez, Laurent Sifre, George van den Driessche, Julian Schrittwieser, et al. "Mastering the Game of Go with Deep Neural Networks and Tree Search.” Nature 529, no. 7587 (January 27, 2016): 484-489. doi:10.1038/nature16961.

[17] Socher, Richard, John Bauer, Christopher D. Manning, and Andrew Y. Ng. "Parsing with compositional vector grammars." In Proceedings of the 51st Annual Meeting of the Association for Computational Linguistics (Volume 1: Long Papers), (2013): 455-465.

[18] Chong, Eunsuk, Chulwoo Han, and Frank C. Park. "Deep Learning Networks for Stock Market Analysis and Prediction: Methodology, Data Representations, and Case Studies.” Expert Systems with Applications 83 (October 2017): 187-205. doi:10.1016/j.eswa.2017.04.030.

[19] Shih, Shun-Yao, Fan-Keng Sun, and Hung-yi Lee. "Temporal Pattern Attention for Multivariate Time Series Forecasting." Machine Learning 108, no. 8-9 (June 11, 2019): 1421-1441. doi:10.1007/s10994-019-05815-0. 
[20] Hoseinzade, Ehsan, and Saman Haratizadeh. "CNNpred: CNN-Based Stock Market Prediction Using a Diverse Set of Variables.” Expert Systems with Applications 129 (September 2019): 273-285. doi:10.1016/j.eswa.2019.03.029.

[21] Hochreiter, Sepp, and Jürgen Schmidhuber. "Long Short-Term Memory.” Neural Computation 9, no. 8 (November 1, 1997): 1735-1780. doi:10.1162/neco.1997.9.8.1735.

[22] Yadav, Anita, C K Jha, and Aditi Sharan. “Optimizing LSTM for Time Series Prediction in Indian Stock Market.” Procedia Computer Science 167 (2020): 2091-2100. doi:10.1016/j.procs.2020.03.257.

[23] Yuan, Xiaofeng, Lin Li, and Yalin Wang. "Nonlinear Dynamic Soft Sensor Modeling With Supervised Long Short-Term Memory Network." IEEE Transactions on Industrial Informatics 16, no. 5 (May 2020): 3168-3176. doi:10.1109/tii.2019.2902129.

[24] Nabipour, M., P. Nayyeri, H. Jabani, A. Mosavi, E. Salwana, and Shahab S. "Deep Learning for Stock Market Prediction.” Entropy 22, no. 8 (July 30, 2020): 840. doi:10.3390/e22080840.

[25] Kamalov, Firuz. "Forecasting Significant Stock Price Changes Using Neural Networks." Neural Computing and Applications 32, no. 23 (May 4, 2020): 17655-17667. doi:10.1007/s00521-020-04942-3.

[26] Liu, Hui, and Zhihao Long. “An Improved Deep Learning Model for Predicting Stock Market Price Time Series.” Digital Signal Processing 102 (July 2020): 102741. doi:10.1016/j.dsp.2020.102741.

[27] Marblestone, Adam H., Greg Wayne, and Konrad P. Kording. "Toward an Integration of Deep Learning and Neuroscience." Frontiers in Computational Neuroscience 10 (September 14, 2016). doi:10.3389/fncom.2016.00094.

[28] Zhong, Xiao, and David Enke. "Predicting the Daily Return Direction of the Stock Market Using Hybrid Machine Learning Algorithms.” Financial Innovation 5, no. 1 (June 15, 2019). doi:10.1186/s40854-019-0138-0.

[29] Lu, Wenjie, Jiazheng Li, Yifan Li, Aijun Sun, and Jingyang Wang. "A CNN-LSTM-Based Model to Forecast Stock Prices." Edited by Abd E. I.-Baset Hassanien. Complexity 2020 (November 23, 2020): 1-10. doi:10.1155/2020/6622927.

[30] Mahmoud, Amal, and Ammar Mohammed. “A Survey on Deep Learning for Time-Series Forecasting.” Machine Learning and Big Data Analytics Paradigms: Analysis, Applications and Challenges (December 15, 2020): 365-392. doi:10.1007/978-3-03059338-4_19.

[31] Mónica, Julio C., Patricia Melin, and Daniela Sánchez. "Genetic Optimization of Ensemble Neural Network Architectures for Prediction of COVID-19 Confirmed and Death Cases." Studies in Computational Intelligence (2021): 85-98. doi:10.1007/9783-030-68776-2_5.

[32] Liu, Zhuoyi, Ryoichi Hara, and Hiroyuki Kita. "Hybrid Forecasting System Based on Data Area Division and Deep Learning Neural Network for Short-Term Wind Speed Forecasting." Energy Conversion and Management 238 (June 2021): 114136. doi:10.1016/j.enconman.2021.114136.

[33] Vadicharla, Giridhar, and Pushpa Sharma. "An Approach of Optimization Techniques for History Matching and Production Forecasting." New Ideas Concerning Science and Technology Vol. 12 (May 1, 2021): 123-140. doi:10.9734/bpi/nicst/v12/7815d.

[34] Zhang, Zhenwu, and Xiantao Liu. "Study on Optimal Operation of Natural Gas Pipeline Network Based on Improved Genetic Algorithm.” Advances in Mechanical Engineering 9, no. 8 (August 2017): 168781401771598. doi:10.1177/1687814017715981.

[35] Katoch, Sourabh, Sumit Singh Chauhan, and Vijay Kumar. "A Review on Genetic Algorithm: Past, Present, and Future." Multimedia Tools and Applications 80, no. 5 (October 31, 2020): 8091-8126. doi:10.1007/s11042-020-10139-6.

[36] Qu, Zhijian, Hanxin Liu, Zixiao Wang, Juan Xu, Pei Zhang, and Han Zeng. "A Combined Genetic Optimization with AdaBoost Ensemble Model for Anomaly Detection in Buildings Electricity Consumption.” Energy and Buildings 248 (October 2021): 111193. doi:10.1016/j.enbuild.2021.111193.

[37] Doewes, Rumi Iqbal, Rajit Nair, and Tripti Sharma. "Diagnosis of COVID-19 through Blood Sample Using Ensemble Genetic Algorithms and Machine Learning Classifier." World Journal of Engineering ahead-of-print, no. ahead-of-print (July 1, 2021). doi:10.1108/wje-03-2021-0174.

[38] Gao, Yiping, Liang Gao, Xinyu Li, and Cuiyu Wang. "A Genetic Algorithm-Based Ensemble Convolutional Neural Networks for Defect Recognition with Small-Scale Samples.” Advances in Swarm Intelligence (2021): 390-398. doi:10.1007/978-3-03078743-1_35.

[39] Leung, Kathy, Joseph T. Wu, and Gabriel M. Leung. "Real-Time Tracking and Prediction of COVID-19 Infection Using Digital Proxies of Population Mobility and Mixing.” Nature Communications 12, no. 1 (March 8, 2021). doi:10.1038/s41467-02121776-2.

[40] Kuo, Cheng-Pin, and Joshua S. Fu. "Evaluating the Impact of Mobility on COVID-19 Pandemic with Machine Learning Hybrid Predictions." Science of The Total Environment 758 (March 2021): 144151. doi:10.1016/j.scitotenv.2020.144151. 